\title{
Áreas de alta mortalidad perinatal debida a anomalías congénitas: análisis de estadísticas vitales, Colombia, 1999-2008
}

\author{
Sandra Patricia Misnaza1', Claudia Patricia Roncancio², Isabel Cristina Peña ${ }^{3}$, Franklin Edwin Prieto ${ }^{4}$ \\ 1 Programa de Entrenamiento en Epidemiología de Campo, Colombia, Grupo de Investigación en Epidemiología \\ Aplicada, Instituto Nacional de Salud, Bogotá, D.C., Colombia \\ 2 Programa de Entrenamiento en Epidemiología de Campo, Colombia, Grupo de Investigación en Epidemiología \\ Aplicada, Pontificia Universidad Javeriana, Bogotá, D.C., Colombia \\ 3 Corporación Nuestra IPS, Fusagasugá, Colombia \\ 4 Programa de Entrenamiento en Epidemiología de Campo, Colombia, Grupo de Investigación en Epidemiología \\ Aplicada, Training Programs in Epidemiology and Public Health Interventions Network (TEPHINET), Bogotá, D.C., \\ Colombia
}

Introducción. Durante 2012, las anomalías congénitas fueron la causa de $13 \%$ de las muertes en menores de 28 días a nivel mundial y, en Colombia constituyeron la segunda causa de mortalidad infantil.

Objetivo. Determinar la distribución geográfica de la mortalidad perinatal por anomalías congénitas en Colombia entre 1999 y 2008.

Materiales y métodos. Se hizo un estudio descriptivo revisando los certificados de defunción de Colombia. La muerte perinatal se definió como muerte fetal y no fetal en niños con un peso de 500 g o más y 28 o menos días de edad, y las anomalías congénitas, como causa básica de la muerte (Clasificación Internacional de Enfermedades - CIE10: Q000 a Q999). La proyección nacional de nacimientos se tomó como el denominador de las tasas específicas. Se calcularon los percentiles para analizar las áreas de alta mortalidad (percentil de 90 o más).

Resultados. Se encontraron 22.361 muertes perinatales por anomalías congénitas. Antioquia, Caldas, Risaralda, Huila, Quindío, Bogotá, Valle del Cauca y Guainía superaron, en promedio, el percentil 90 durante los diez años de estudio. Los municipios con mayores tasas de mortalidad fueron: Giraldo, Ciudad Bolívar, Riosucio, Liborina, Supía, Alejandría, Sopetrán, San Jerónimo, Santa Fe de Antioquia y Marmato, en donde oscilaron entre 205,81 y 74,18 por 10.000 nacimientos. Las tasas de mortalidad perinatal por 10.000 nacidos vivos fueron de 28,1 para el grupo de malformaciones del sistema circulatorio; de 13,7 para anomalías del sistema nervioso central, y de 7,0 para anomalías cromosómicas.

Conclusión. La alta mortalidad perinatal por anomalías congénitas en la región andina requiere acciones urgentes de investigación sobre los posibles riesgos y medidas de prevención.

Palabras clave: anomalías congénitas, mortalidad, mortalidad fetal, mortalidad infantil, estadísticas vitales, Colombia.

doi: http://dx.doi.org/10.7705/biomedica.v36i3.2867

\section{Geographic distribution of perinatal mortality due to congenital malformations in Colombia, 1999-2008: An analysis of vital statistics data}

Introduction: During 2012, 13\% of the deaths worldwide in children under the age of 28 days were due to congenital malformations. In Colombia, congenital malformations are the second leading cause of infant mortality.

Objective: To determine the geographical distribution of extended perinatal mortality due to congenital malformations in Colombia between 1999 and 2008.

Materials and methods: We conducted a cross-sectional study. We revised all death certificates issued between 1999 and 2008. We defined perinatal mortality as fetal or non-fetal deaths within the first 28 days after delivery in children with body weight $\geq 500$ grams, and congenital malformations according to ICD-10 diagnostic codes Q000 - Q999. The annual birth projection was used as the denominator. We defined high mortality areas due to congenital malformations as those in the $90^{\text {th }}$ percentile.

Results: We recorded 22,361 perinatal deaths due to congenital malformations. The following provinces exceeded the 90th perinatal mortality percentile: Antioquia, Caldas, Risaralda, Huila, Quindío, Bogotá, Valle del Cauca and Guainía. Among the municipalities, the highest perinatal mortality rates were found

\section{Contribución de los autores:}

Todos los autores participaron en el proceso de solicitud de la información ante la entidad correspondiente, en su consolidación y análisis, y en la redacción del manuscrito. 
in Giraldo, Ciudad Bolívar, Riosucio, Liborina, Supía, Alejandría, Sopetrán, San Jerónimo, Santa Fe de Antioquia and Marmato (205.81 and 74.18 per 10.000 live births). The perinatal mortality rate due to malformations of the circulatory system was 28.1 per 10.000 live births, whereas the rates for central nervous system defects and chromosomal abnormalities were 13.7 and 7.0, respectively.

Conclusions: The Andean region showed high perinatal mortality rates due to congenital malformations. There is an urgent need to identify possible risk factors of perinatal mortality and implement successive prevention programs in that particular region.

Key words: Congenital abnormalities, mortality, fetal mortality, infant mortality, vital statistics, Colombia. doi: http://dx.doi.org/10.7705/biomedica.v36i3.2867

Las tasas de mortalidad infantil, enfermedades crónicas y discapacidad por anomalías, trastornos o malformaciones congénitas son elevadas (1) y están asociadas con los abortos espontáneos. Se ha establecido que algunas anomalías congénitas tienen origen genético, infeccioso o ambiental, aunque en la mayoría de los casos resulta difícil determinar su causa (2). Estas anomalías pueden ocasionar discapacidades crónicas que tienen un gran impacto en los afectados, sus familias, los sistemas de salud y la sociedad (1-3).

De las 2'019.320 muertes registradas a nivel mundial durante 2012, 13 \% se debió a anomalías congénitas en niños menores de 28 días (4). En la región de las Américas, las malformaciones congénitas, las deformidades y las anomalías cromosómicas representaron $20 \%$ de la mortalidad en menores de un año (4). En Suramérica, la tasa de anomalías por 10.000 nacidos vivos responde sobre todo a los defectos cardiacos, los de cierre del tubo neural, el síndrome de Down, el labio leporino con paladar hendido o sin él y los defectos de la pared abdominal (5).

Durante 2010, en Colombia, las anomalías congénitas, las deformidades y las alteraciones cromosómicas representaron una tasa de mortalidad por 100.000 habitantes de 6,7 en niños de 1 a 4 años y de 6,4 en niñas del mismo grupo de edad (6). La tasa de mortalidad por estas causas en menores de un año fue de 256 por 100.000 nacidos vivos, es decir, $21 \%$ de la mortalidad en este grupo de edad (7).

El desarrollo e implementación de programas para la detección y la prevención de estas condiciones requieren la determinación de las poblaciones

Correspondencia:

Sandra Patricia Misnaza, Calle 59 A bis № 5-13, apartamento

701, Bogotá, D.C., Colombia

Teléfono: 3154189193

sandramisnaza@yahoo.com

Recibido: 29/05/15; aceptado: 15/03/16 o áreas con riesgo de mortalidad perinatal en Colombia, por lo cual el objetivo de este estudio fue establecer la mortalidad perinatal debida a anomalías congénitas entre 1999 y 2008 , por departamentos y municipios, con base en la información registrada en los certificados de defunción.

\section{Materiales y métodos}

Se revisaron los certificados de defunción como fuente de información secundaria, específicamente los de las muertes fetales en niños con un peso de $500 \mathrm{~g}$ o más $(\mathrm{n}=191.182)$ y las muertes neonatales en niños con edades comprendidas entre los 0 y los 28 días ( $n=75.938$ ), para un total de 267.120 muertes registradas en el sistema de estadísticas vitales del Departamento Nacional de Estadística de Colombia (DANE) entre 1999 y 2008. La muerte fetal se definió como la de los niños con peso de $500 \mathrm{~g}$ o más y, la no fetal, como la de aquellos con edades comprendidas entre los 0 y los 28 días. La definición de muerte por anomalías congénitas se basó en los códigos Q000 a Q999 de la Clasificación Internacional de Enfermedades - CIE-10.

En el certificado de defunción se enumeran varios tipos de causas de muerte: causa básica, directa, antecedente y patológica. La causa básica, entendida como la enfermedad o lesión que inició la cadena de acontecimientos patológicos que condujeron directamente a la muerte, se tomó como referente en la definición de caso (8).

Las variables analizadas incluyeron la fecha de nacimiento del fallecido, su lugar de residencia habitual (país, departamento y municipio), la edad de la madre y la causa básica de la defunción.

Se evaluó la calidad de los datos con base en su integridad (completitud de los datos en los campos del certificado de defunción) y se verificó la proporción de los campos sin datos en las secciones seleccionadas del certificado de defunción.

La información se desagregó por anomalías congénitas cromosómicas, del sistema circulatorio y del sistema nervioso central. 


\section{Análisis estadístico}

Las tasas de mortalidad se calcularon utilizando la proyección nacional de nacimientos anuales como denominador, para luego promediar los valores de los diez años de estudio. La mortalidad por áreas en cada departamento y municipio de residencia se expresó en percentiles (25, 50, 75 y 90). Las tasas de mortalidad perinatal se identificaron mediante una gradación de colores equivalente a los percentiles (blanco: percentil $\leq 25$; gris claro: percentil 50; gris medio: percentil 75, y gris oscuro: percentil $\geq 90$ ), con el fin de señalar los departamentos con las mayores y menores tasas de mortalidad (figura 1). Esto permitió obtener un panorama para priorizar las áreas geográficas que requerirían más investigación, y para planificar las acciones de intervención y prevención a corto y mediano plazo.

Los mapas sobre la mortalidad en cada año se hicieron con el programa Arcgis $9.3^{\circledR}$ y se graficaron con base en los promedios de las tasas de mortalidad durante los diez años de estudio.

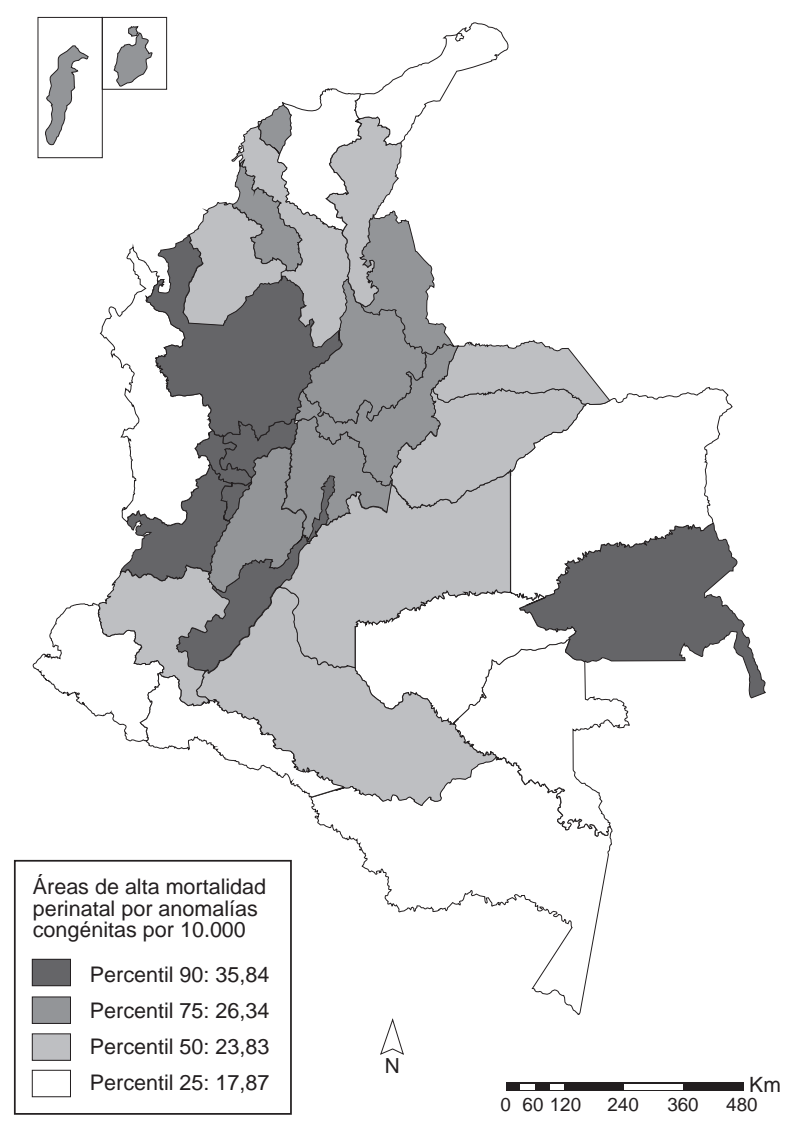

Figura 1. Áreas de alta mortalidad perinatal por anomalías congénitas y ente territorial, Colombia, 1999-2008.

\section{Consideraciones éticas}

En el diseño del estudio se tuvieron en cuenta los principios metodológicos para salvaguardar el interés de la ciencia y el respeto a los derechos de las personas. Las bases de datos sobre defunciones no contenían la identificación de los individuos ni de sus padres; no hubo ninguna intervención en los sujetos, no se violó el derecho a la privacidad y los resultados se presentan de manera agrupada. Según la norma nacional (Resolución 008430 de 1993), este tipo de estudio se considera de riesgo mínimo.

\section{Resultados}

De 1999 a 2008, se identificaron 267.120 muertes perinatales en Colombia, de las cuales 22.361 se debieron a anomalías congénitas.

La integridad de la información sobre las variables de análisis fue del $100 \%$, a excepción de los datos sobre la edad de la madre, con $88 \%$.

Las anomalías del sistema circulatorio fueron responsables de $32,3 \%$ de las muertes; las del sistema nervioso central, de $16 \%$, y las congénitas de origen cromosómico, de $8 \%$; estos tres grupos representaron 56,3\% del total de muertes por estas causas. Las causas de las demás muertes perinatales fueron, en su orden, otra anomalía congénita $(26,2 \%)$, anomalías del sistema músculo-esquelético (5,6\%), del sistema digestivo (4,9\%), del sistema respiratorio (4\%) y del sistema urinario (2,9\%). La tasa promedio de mortalidad perinatal por 10.000 nacidos vivos, durante los diez años, fue de 26,18 (cuadro 1), la tasa promedio de mortalidad fetal fue de 8,47 y la tasa promedio de mortalidad neonatal fue de 17,39 de por 10.000 nacidos vivos.

En el análisis de la situación geográfica, los promedios de los percentiles por 10.000 nacidos vivos durante los diez años de estudio fueron los siguientes: $\leq 17,87$ para el percentil $25 ; 23,83$ para el percentil 50; 26,34 para el percentil $75, y>35,84$ para el percentil 90.

El análisis por departamentos evidenció que en Antioquia hubo más de 35,84 casos por 10.000 nacidos vivos (se superó el percentil 90), seguido por los departamentos de Risaralda, Quindío y Guainía, los cuales superaron el percentil 90 durante cuatro años, mientras que Caldas tuvo altas tasas durante tres años; en Huila y San Andrés se mantuvieron estables durante dos años, y Valle del Cauca, Vichada, Cesar, Sucre y Santander sobrepasaron este valor durante uno de los años (figura 1). 
Cuadro 1. Tasas de mortalidad perinatal por anomalías congénitas por 100.000 nacidos vivos por ente territorial, Colombia, 19992008

\begin{tabular}{|c|c|c|c|c|c|c|c|c|c|c|c|}
\hline Ente territorial & 1999 & 2000 & 2001 & 2002 & 2003 & 2004 & 2005 & 2006 & 2007 & 2008 & $\begin{array}{c}\text { Tasa } \\
\text { promedio }\end{array}$ \\
\hline Tasa de mortalidad nacional & 22,35 & 26,2 & 25,53 & 25,08 & 26,01 & 24,06 & 26,43 & 27 & 31,76 & 24,14 & 26,18 \\
\hline Antioquia & 68,14 & 43,5 & 34,19 & 34,35 & 39,81 & 34,78 & 40,25 & 38,95 & 55,39 & 29,69 & 39,25 \\
\hline Caldas & 65,66 & 34,26 & 34,12 & 28 & 27,07 & 32,5 & 29,37 & 27,3 & 72,36 & 71,76 & 38,66 \\
\hline Risaralda & 53,73 & 32,49 & 37,94 & 32,54 & 39,55 & 28,67 & 24,25 & 29,35 & 41,54 & 35,73 & 34,07 \\
\hline Huila & 52,64 & 49,89 & 53,27 & 28,03 & 27,5 & 20,13 & 30,03 & 28,46 & 31,22 & 18,03 & 32,16 \\
\hline Quindío & 54,44 & 43,28 & 40,26 & 30,09 & 17,38 & 40,83 & 20,81 & 32,35 & 25,69 & 20,4 & 31,08 \\
\hline Bogotá, D.C. & 45,37 & 24,33 & 26,02 & 26,13 & 28,35 & 29,99 & 29,71 & 29,45 & 37,91 & 22,79 & 28,46 \\
\hline Valle del Cauca & 54,69 & 26,75 & 26,95 & 29,46 & 31,77 & 21,1 & 26,13 & 27,4 & 23,11 & 27,73 & 27,5 \\
\hline Guainía & 65,65 & 21,46 & 10,68 & 10,79 & 43,57 & 0 & 45,25 & 0 & 77,95 & 0 & 26,49 \\
\hline Santander & 51,29 & 29,49 & 26,21 & 23,98 & 20,05 & 23,34 & 22,17 & 30,13 & 28,47 & 24,89 & 26,34 \\
\hline Sucre & 25,67 & 14,05 & 19,09 & 23,65 & 25,09 & 27,5 & 34,93 & 29,26 & 29,29 & 37,61 & 25,71 \\
\hline Tolima & 43,98 & 32,7 & 32,61 & 27,72 & 24,37 & 22,01 & 21,73 & 20,93 & 24,2 & 21,4 & 25,31 \\
\hline Atlántico & 39,1 & 24,59 & 27,09 & 28,9 & 29,12 & 23,48 & 22,6 & 26,78 & 26,53 & 24,93 & 25,29 \\
\hline Norte de Santander & 49,05 & 27,63 & 31,8 & 23,18 & 19,75 & 17,38 & 20,3 & 20,69 & 32,48 & 31,38 & 25,26 \\
\hline San Andrés & 55,4 & 7,34 & 22,73 & 38,88 & 31,47 & 15,66 & 23,13 & 23,29 & 39,09 & 15,71 & 25,06 \\
\hline Cundinamarca & 29,68 & 20,19 & 19,21 & 21,51 & 23,02 & 21,46 & 24,85 & 39,73 & 38,16 & 17,42 & 24,18 \\
\hline Boyacá & 35,18 & 27,49 & 23,54 & 26,68 & 24,02 & 22,88 & 26,19 & 25,76 & 24,66 & 18,26 & 24,02 \\
\hline Caquetá & 27,22 & 24,21 & 23,4 & 25,27 & 27,23 & 25,69 & 30,38 & 23,45 & 20,95 & 23,92 & 23,83 \\
\hline Cauca & 36,03 & 21,14 & 20,65 & 18 & 24,95 & 18,1 & 24,45 & 19,32 & 27,84 & 24,74 & 22,09 \\
\hline Cesar & 23,05 & 16,39 & 19,54 & 18,81 & 34,78 & 23,43 & 22,15 & 23,64 & 28,67 & 20,68 & 22,02 \\
\hline Arauca & 44,59 & 12,19 & 18,33 & 22,74 & 17,87 & 21,71 & 12,45 & 25,02 & 28,23 & 40,52 & 22 \\
\hline Córdoba & 31,63 & 22,89 & 23,77 & 19,09 & 21,03 & 21,01 & 24,02 & 17,3 & 20,53 & 21,65 & 21,07 \\
\hline Casanare & 30,11 & 14,03 & 11,31 & 9,92 & 24,18 & 24,22 & 25,63 & 25,69 & 27,16 & 25,75 & 20,37 \\
\hline Meta & 36,14 & 15,17 & 14,47 & 25,53 & 20,76 & 19,61 & 27,48 & 25,32 & 19,47 & 13,85 & 20,03 \\
\hline Bolívar & 33,45 & 16,79 & 19,31 & 22,6 & 18,73 & 17,38 & 20,45 & 19,96 & 15,14 & 17,29 & 18,76 \\
\hline Magdalena & 24,25 & 17,28 & 12,28 & 16,74 & 16,57 & 16,59 & 27,36 & 20,62 & 21,52 & 19,34 & 17,87 \\
\hline Amazonas & 0 & 14,33 & 9,53 & 19,08 & 4,8 & 19,53 & 19,93 & 10,08 & 40 & 14,11 & 15,14 \\
\hline Putumayo & 12,77 & 11,38 & 12,88 & 13,21 & 18,28 & 12,27 & 15,87 & 27,22 & 16,25 & 15,07 & 15,1 \\
\hline Guaviare & 14,45 & 25,13 & 17,87 & 21,34 & 7,1 & 21,21 & 10,66 & 7,02 & 20,91 & 11 & 14,85 \\
\hline Nariño & 23,06 & 12,52 & 12,51 & 12,58 & 13,31 & 17,05 & 10,18 & 14 & 22,43 & 12,63 & 13,84 \\
\hline La Guajira & 32,47 & 11,03 & 9,31 & 14,31 & 11,52 & 16,16 & 18,63 & 14,96 & 13,21 & 13,7 & 13,45 \\
\hline Chocó & 10,3 & 11,67 & 15,4 & 11 & 6,61 & 12,5 & 14,03 & 14,72 & 13,96 & 16,17 & 12,13 \\
\hline Vaupés & 0 & 24,19 & 23,98 & 15,92 & 7,97 & 8,06 & 0 & 8,17 & 16,37 & 14,14 & 12,1 \\
\hline Vichada & 61,2 & 6,01 & 11,79 & 17,4 & 0 & 0 & 0 & 0 & 11,26 & 0 & 7,42 \\
\hline
\end{tabular}

Los municipios con mayores tasas de mortalidad por 10.000 nacidos vivos fueron: Giraldo, Ciudad Bolívar, Liborina, Alejandría, Sopetrán, San Jerónimo y Santa Fe de Antioquia en Antioquia, con 205,81, 134,83, 128,21, 93,98, 89,73, 87,34 y 85,08, respectivamente, y Riosucio, Supía y Marmato en Caldas, con 132,18, 101,79 y 74,18, respectivamente.

De las muertes perinatales, 18,8 \% correspondió a madres menores de 18 años y, 14,2\%, a madres mayores de 35 años. Los departamentos con mayor proporción de muertes debido a anomalías congénitas en hijos de madres mayores de 35 años fueron San Andrés (21\%), Caldas (18\%) y Antioquia (17\%).

Las anomalías con mayores tasas de mortalidad perinatal por 10.000 nacidos vivos fueron las del sistema circulatorio $(28,1)$, las del sistema nervioso central $(13,7)$ y las cromosómicas no clasificadas en otra parte $(7,0)$.

Las tasas de mortalidad fetal por 10.000 nacidos vivos para el grupo de anomalías del sistema circulatorio fue de 1,74; para el de anomalías del sistema nervioso central, 5,39, y para el de anomalías de origen cromosómico, 5,71.

Las tasas de mortalidad neonatal por 10.000 nacidos vivos para el grupo de anomalías del sistema circulatorio fue de 26,33; para el de anomalías del sistema nervioso central, 8,27, y para el de anomalías cromosómicas, 1,27.

Entre 1999 y 2008 las mayores tasas de mortalidad perinatal por anomalías congénitas del sistema circulatorio, del sistema nervioso central y las cromosómicas, se presentaron en Antioquia, Cesar, Santander, y Boyacá (cuadros 2, 3 y 4). 
En el grupo de anomalías del sistema nervioso central, los defectos del tubo neural representaron $51,8 \%$ de las muertes en los diez años del estudio, de las cuales $76,5 \%$ se debió a anencefalia, $13,8 \%$ a espina bífida y 9,8 \% a encefalocele. La tasa nacional promedio por defectos del tubo neural fue de 2,1 por 10.000 nacidos vivos, pero tendió a disminuir a partir de 2005; Caquetá, Guainía, Quindío, Córdoba, Vichada, Norte de Santander, Antioquia, Risaralda, Huila, Santander, Tolima,
Caldas, Atlántico, Magdalena, Arauca, Casanare, La Guajira, Putumayo, Boyacá y Sucre superaron la tasa promedio de mortalidad nacional (cuadro 5).

\section{Discusión}

La tasa de mortalidad perinatal promedio debida a anomalías congénitas por 10.000 nacidos vivos en los diez años de estudio fue de 26,18 y la tasa promedio de mortalidad neonatal fue de 17,39 por 10.000 nacidos vivos, siendo superior a la

Cuadro 2. Lugares con las mayores tasas de mortalidad perinatal por anomalías congénitas cromosómicas, Colombia, 1999-2008

\begin{tabular}{lccccc}
\hline Departamento & Municipio & $\begin{array}{c}\text { Tasa municipal } \\
\text { promedio } \\
\text { por } \mathbf{1 0 . 0 0 0}\end{array}$ & Anomalía congénita & $\begin{array}{c}\text { n } \\
\text { Tasa promedio de } \\
\text { anomalías congénitas } \\
\text { por 10.000 }\end{array}$ \\
\hline Antioquia & Giraldo & 121,07 & Anomalía cromosómica, NE & 10 & 121,07 \\
Antioquia & Ciudad Bolívar & 105,46 & Anomalía cromosómica, NE & 79 & 105,46 \\
Antioquia & Liborina & 83,33 & Anomalía cromosómica, NE & 13 & 83,33 \\
Antioquia & Sopetrán & 66,47 & Anomalía cromosómica, NE & 20 & 66,47 \\
Cesar & La Jagua de lbirico & 60,78 & Anomalía cromosómica, NE & 42 & 60,78 \\
\hline
\end{tabular}

NE: no especificada

Cuadro 3. Lugares con las mayores tasas de mortalidad perinatal por anomalías congénitas del sistema circulatorio, Colombia, 1999-2008

\begin{tabular}{lccccc}
\hline Departamento & Municipio & $\begin{array}{c}\text { Tasa promedio } \\
\text { por 10.000 }\end{array}$ & Anomalía congénita & $\begin{array}{c}\text { n } \\
\text { Tasa promedio de } \\
\text { anomalías congénitas } \\
\text { por 10.000 }\end{array}$ \\
\hline Santander & Palmas del Socorro & 60,48 & Malformación congénita del corazón, NE & 3 & 60,48 \\
Santander & Macaravita & 37,38 & Malformación congénita del corazón, NE & 2 & 37,38 \\
Santander & San Joaquín & 32,36 & Malformación congénita del corazón, NE & 1 & 16,18 \\
& & & Otras malformaciones congénitas de la & 1 & 16,18 \\
Boyacá & Pachavita & 29,46 & Málvula pulmonar & Malformación congénita del corazón, NE & 2 \\
Santander & Onzaga & 27,45 & Malformación congénita del corazón, NE & 4 & 29,46 \\
\hline
\end{tabular}

NE: no especificada

Cuadro 4. Lugares con las mayores tasas de mortalidad perinatal por anomalías congénitas del sistema nervioso central, Colombia, 1999-2008

\begin{tabular}{|c|c|c|c|c|c|}
\hline Departamento & Municipio & $\begin{array}{l}\text { Tasa promedio } \\
\text { por } 10.000\end{array}$ & Anomalía congénita & $\mathbf{n}$ & $\begin{array}{c}\text { Tasa promedio de } \\
\text { anomalías congénitas } \\
\text { por } 10.000\end{array}$ \\
\hline \multirow[t]{3}{*}{ Antioquia } & Giraldo & 36,32 & Malformación congénita del sistema nervioso, NE & 1 & 12,11 \\
\hline & & & Anencefalia & 1 & 12,11 \\
\hline & & & Otras anomalías hipoplásicas del encéfalo & 1 & 12,11 \\
\hline \multirow[t]{2}{*}{ Boyacá } & Sativanorte & 34,84 & Anencefalia & 1 & 17,42 \\
\hline & & & Encefalocele, NE & 1 & 17,42 \\
\hline \multirow[t]{4}{*}{ Antioquia } & Granada & 25,62 & Hidrocéfalo congénito, NE & 3 & 12,81 \\
\hline & & & Otras anomalías hipoplásicas del encéfalo & 1 & 4,27 \\
\hline & & & Malformación congénita del sistema nervioso, NE & 1 & 4,27 \\
\hline & & & Anencefalia & 1 & 4,27 \\
\hline Boyacá & Iza & 23,70 & Anencefalia & 1 & 23,70 \\
\hline \multirow{2}{*}{ Boyacá } & Tota & 20,28 & Malformación congénita del sistema nervioso, NE & 1 & 10,14 \\
\hline & & & Anencefalia & 1 & 10,14 \\
\hline
\end{tabular}

NE: no especificada 
mortalidad neonatal por estas causas de Canadá $(1,1$ por 1.000$)$ y Estados Unidos (1,1 por 1.000), e inferior a la tasa de mortalidad de Argentina (2,4 por 1.000 nacidos vivos), Brasil (2,2 por $1.000)$, Chile (2,2 por 1.000) y Costa Rica (2,1 por 1.000) (3).

Los departamentos con las mayores tasas de mortalidad perinatal debidas a anomalías congénitas fueron, principalmente, los de la región andina, con la excepción de Guainía, departamento localizado en la región amazónica del país. La distribución de los municipios de mayor mortalidad fue similar, pues los diez municipios con mayores tasas de mortalidad son de la región andina, la cual concentra la mayoría de la población colombiana (9) y comprende grandes centros hidrográficos del país (10), tiene una gran variedad de climas y es una zona que ha recibido cenizas volcánicas, por lo cual tiene un gran potencial agrícola (11).
En algunos estudios se ha reportado que una gran proporción de los casos de muerte perinatal se registran sin diagnóstico porque ocurre en los hogares, y las madres han recibido poca o ninguna atención calificada. Se sabe, sin embargo, que, incluso cuando se cuenta con la mejor tecnología, en 20 a $50 \%$ de los casos de mortinatos no se registra la causa de la muerte (12), lo cual podría explicar la menor distribución registrada en los departamentos de las regiones Amazónica y Caribe en este estudio.

En otros estudios se ha evidenciado una estrecha asociación entre la edad materna avanzada y las anomalías congénitas, principalmente las de origen cromosómico $(13,14)$, así como un mayor riesgo de cierto tipo de anomalías no cromosómicas en mujeres gestantes muy jóvenes (15). En general, este mayor riesgo de mortalidad perinatal se registra en las edades extremas (16); sin embargo,

Cuadro 5. Tasas de mortalidad perinatal por 10.000 nacidos vivos debida a defectos del tubo neural, Colombia, 1999-2008

\begin{tabular}{|c|c|c|c|c|c|c|c|c|c|c|c|}
\hline Ente territorial & 1999 & 2000 & 2001 & 2002 & 2003 & 2004 & 2005 & 2006 & 2007 & 2008 & Promedio \\
\hline Tasa de mortalidad nacional & 2,1 & 2,5 & 2,0 & 2,5 & 2,4 & 2,2 & 2,1 & 1,8 & 1,9 & 1,5 & 2,1 \\
\hline Caquetá & 3,4 & 2,6 & 2,6 & 5,2 & 4,4 & 3,5 & 4,5 & 2,7 & 4,6 & 1,8 & 3,5 \\
\hline Guainía & 0,0 & 0,0 & 0,0 & 0,0 & 10,9 & 22,1 & 0,0 & 0,0 & 0,0 & 0,0 & 3,3 \\
\hline Quindío & 3,9 & 4,2 & 3,4 & 3,5 & 2,3 & 6,8 & 2,2 & 1,1 & 2,1 & 3,2 & 3,3 \\
\hline Córdoba & 6,8 & 3,6 & 1,5 & 2,5 & 4,3 & 2,4 & 5,1 & 1,1 & 0,8 & 2,5 & 3,0 \\
\hline Vichada & 24,5 & 0,0 & 5,9 & 0,0 & 0,0 & 0,0 & 0,0 & 0,0 & 0,0 & 0,0 & 3,0 \\
\hline Norte de Santander & 6,5 & 3,3 & 5,7 & 1,7 & 1,8 & 1,5 & 3,1 & 2,4 & 2,1 & 2,0 & 3,0 \\
\hline Antioquia & 6,9 & 3,2 & 2,8 & 3,5 & 2,6 & 2,6 & 2,0 & 1,9 & 2,5 & 2,0 & 3,0 \\
\hline Risaralda & 4,6 & 3,1 & 2,6 & 3,3 & 3,4 & 4,0 & 2,0 & 3,9 & 1,3 & 0,6 & 2,9 \\
\hline Huila & 4,8 & 3,4 & 2,6 & 2,6 & 1,8 & 3,6 & 1,3 & 2,3 & 3,2 & 2,7 & 2,8 \\
\hline Santander & 4,4 & 2,8 & 2,1 & 3,9 & 2,3 & 2,9 & 1,5 & 3,0 & 1,8 & 2,1 & 2,7 \\
\hline Tolima & 5,4 & 3,8 & 1,8 & 2,5 & 3,3 & 1,8 & 1,5 & 1,1 & 3,4 & 1,9 & 2,7 \\
\hline Caldas & 4,6 & 3,4 & 3,4 & 3,4 & 2,3 & 2,9 & 2,9 & 1,2 & 1,2 & 0,0 & 2,5 \\
\hline Atlántico & 4,9 & 3,2 & 1,6 & 3,2 & 2,3 & 2,5 & 1,8 & 1,8 & 2,1 & 1,6 & 2,5 \\
\hline Magdalena & 4,2 & 2,3 & 1,3 & 4,0 & 1,4 & 1,4 & 3,6 & 1,4 & 2,6 & 2,2 & 2,4 \\
\hline Arauca & 3,0 & 1,5 & 6,1 & 0,0 & 4,5 & 2,9 & 0,0 & 2,8 & 0,0 & 2,9 & 2,4 \\
\hline Casanare & 2,7 & 5,6 & 0,0 & 1,4 & 4,3 & 0,0 & 0,0 & 1,4 & 1,4 & 5,7 & 2,3 \\
\hline La Guajira & 4,8 & 0,6 & 0,5 & 3,5 & 2,9 & 2,4 & 2,9 & 1,9 & 1,4 & 1,3 & 2,2 \\
\hline Putumayo & 2,1 & 2,3 & 2,3 & 0,0 & 2,4 & 1,2 & 3,7 & 4,9 & 1,3 & 1,3 & 2,2 \\
\hline Boyacá & 2,1 & 2,6 & 2,2 & 1,5 & 1,5 & 3,6 & 0,0 & 4,1 & 2,1 & 1,7 & 2,1 \\
\hline Sucre & 2,1 & 0,6 & 0,0 & 0,6 & 2,4 & 1,8 & 7,1 & 1,2 & 2,4 & 3,0 & 2,1 \\
\hline Valle del Cauca & 2,5 & 2,1 & 1,8 & 2,9 & 2,5 & 1,3 & 2,1 & 1,5 & 1,7 & 1,7 & 2,0 \\
\hline Cesar & 2,6 & 1,3 & 1,3 & 1,7 & 1,3 & 2,7 & 3,1 & 3,1 & 1,3 & 1,3 & 2,0 \\
\hline Cauca & 3,2 & 2,3 & 1,4 & 1,8 & 2,9 & 1,1 & 1,1 & 1,2 & 2,0 & 1,6 & 1,8 \\
\hline Bolívar & 2,6 & 2,0 & 2,0 & 2,5 & 2,3 & 1,0 & 1,4 & 1,7 & 1,7 & 0,5 & 1,8 \\
\hline Bogotá, D.C. & 3,3 & 2,3 & 1,4 & 1,4 & 2,2 & 1,8 & 1,6 & 1,2 & 1,5 & 0,3 & 1,7 \\
\hline Chocó & 1,5 & 1,5 & 2,9 & 2,2 & 1,5 & 2,2 & 0,7 & 0,7 & 2,2 & 1,5 & 1,7 \\
\hline Meta & 1,2 & 0,6 & 1,3 & 0,6 & 2,4 & 2,4 & 3,4 & 2,8 & 0,0 & 1,7 & 1,6 \\
\hline Cundinamarca & 3,0 & 1,5 & 1,7 & 1,3 & 1,1 & 1,8 & 0,9 & 1,8 & 1,6 & 0,4 & 1,5 \\
\hline Vaupés & 0,0 & 0,0 & 0,0 & 8,0 & 0,0 & 0,0 & 0,0 & 0,0 & 0,0 & 7,1 & 1,5 \\
\hline Guaviare & 0,0 & 7,2 & 0,0 & 7,1 & 0,0 & 0,0 & 0,0 & 0,0 & 0,0 & 0,0 & 1,4 \\
\hline Nariño & 1,7 & 1,7 & 0,6 & 1,7 & 1,7 & 1,5 & 0,9 & 0,6 & 1,5 & 0,6 & 1,3 \\
\hline Amazonas & 0,0 & 0,0 & 0,0 & 0,0 & 4,8 & 0,0 & 5,0 & 0,0 & 0,0 & 0,0 & 1,0 \\
\hline Archipiélago de San Andrés & 0,0 & 0,0 & 0,0 & 0,0 & 0,0 & 0,0 & 0,0 & 0,0 & 0,0 & 7,9 & 0,8 \\
\hline
\end{tabular}


en este estudio la mortalidad perinatal causada por anomalías congénitas fue más frecuente en las mujeres entre 20 y 34 años, lo cual se explicaría por su pertenencia al grupo mayoritario de mujeres en edad fértil.

Las anomalías congénitas de los sistemas circulatorio y nervioso central, y las anomalías cromosómicas fueron la causa de 56,3 \% de las muertes por estas condiciones, lo cual es similar a la situación registrada en Estados Unidos entre 1997 y 2007, años en los cuales 60 \% de las muertes correspondió a anomalías del sistema nervioso, cardiovascular y respiratorio (17).

En los municipios de Antioquia se registraron más frecuentemente las anomalías cromosómicas, pero la proporción de nacidos vivos en este departamento es mayor según las proyecciones del Departamento Administrativo Nacional de Estadística (DANE), lo que explicaría el aumento en dicha frecuencia. Según las estadísticas vitales del DANE (18), entre 1999 y 2008, $10 \%$ de los nacimientos nacionales fueron de mujeres gestantes mayores. En Antioquia, $17,1 \%$ de la mortalidad por anomalías congénitas se registró en mujeres mayores de 35 años, lo cual coincidiría con la relación reportada entre edad avanzada de la madre y anomalías de origen cromosómico (13-14,19).

Las tasas más altas de anomalías del sistema circulatorio se presentaron en los municipios de Santander y Boyacá; estas anomalías son las más comunes y representan la mayor causa de mortalidad y morbilidad en el periodo perinatal $(17,20-22)$, pues se estima que afectan de 8 a 10 de cada 1.000 nacidos vivos, aunque la mortalidad es, aproximadamente, 10 veces más frecuente (23). Según el reporte global de defectos congénitos, se estima que en Colombia la prevalencia de anomalías del sistema cardiovascular es, aproximadamente, de 7,7 por 1.000 nacidos vivos, seguidas de las anomalías del sistema osteomuscular (24).

En Colombia, las cardiopatías congénitas son la segunda causa de muerte en menores de un año $(25,26)$ y $34 \%$ de ellas ocurre en el periodo neonatal tardío. En un estudio se determinó que en $80,7 \%$ de las anomalías la intervención del personal de salud, adecuada o inadecuada, modificaba el pronóstico (27). La mortalidad perinatal por anomalías del sistema circulatorio fue de $32,2 \%$, siendo la principal causa de mortalidad en dicho estudio.

Las mayores tasas de anomalías del sistema nervioso central se registraron en municipios de Antioquia y Boyacá, en los cuales se reportó, por lo menos, una muerte cuya causa básica fue la anencefalia; entre las cinco primeras causas básicas de mortalidad perinatal por anomalías del sistema nervioso central, los defectos del tubo neural fueron una constante y representaron un poco más de la mitad de las causas de muerte en este grupo. Es importante recordar que algunas de las manifestaciones de tales anomalías son incompatibles con la vida, o dejan secuelas graves en caso de que el recién nacido sobreviva, lo cual ocasiona un gran impacto psicológico, social y económico para la familia y para la sociedad (28). En este sentido, se ha demostrado que la administración de ácido fólico antes de la gestación puede reducir su prevalencia en 50 a 70 \% (29-31), lo cual constituye una oportunidad para reducir la mortalidad perinatal e infantil. En 1996, se reglamentó en el país la fortificación de la harina de trigo con ácido fólico (32) y algunas industrias también fortifican el arroz por iniciativa propia, situación que podría explicar la tendencia a la disminución en las tasas de mortalidad nacional por defectos del tubo neural a partir de 2005.

Algunas estrategias de salud individual y colectiva reducen la prevalencia de anomalías congénitas, entre ellas, las intervenciones nutricionales, la prevención de enfermedades e infecciones maternas, el control previo a la concepción en madres con enfermedades preexistentes y en casos de exposición profesional y ambiental a teratógenos, así como una especial atención durante el embarazo a mujeres con exposición a factores de riesgo evidentes, como la obesidad y el consumo de tabaco, alcohol o drogas (32). Es por esto que el análisis de las anomalías por áreas de alta mortalidad según los certificados de defunción (33) permite determinar aquellas en donde se deberían evaluar las acciones de salud pública. Además, los factores relacionados con la posible etiología de las anomalías son diferentes según el sistema implicado y la población afectada, pues algunos grupos presentan tasas más altas que otros (34), de manera que el análisis por municipio, departamento y grupo de anomalías permite una mejor aproximación a los factores puntuales que contribuyen a su aparición, para así proponer las medidas de intervención más apropiadas.

En el presente análisis hubo limitaciones por el gran número de casos con anomalías congénitas descritas en el certificado de defunción como "otras" (26,2 \%), lo cual impidió una mayor especificidad. Este proceso de verificación de las causas le corresponde al DANE como entidad 
responsable de la información. La calidad de los datos fue suficiente para agrupar las anomalías por sistemas. Los sesgos de información y de selección se contrarrestaron mediante la verificación de la calidad de la información sobre las causas específicas de muerte por anomalías y su relación con otras reportadas en el certificado de defunción, así como de la concordancia de los datos de edad. Los casos incluidos en el análisis correspondieron a aquellos cuyo registro respondía a la codificación empleada en el estudio.

Se concluyó que en los departamentos y municipios hay áreas con alta mortalidad según el grupo de anomalías congénitas, principalmente en la región andina; Palmas del Socorro, Macaravita, San Joaquín y Onzaga en Santander y Pachavita en Boyacá registraron las mayores tasas de mortalidad por anomalías del sistema circulatorio; Giraldo y Granada en Antioquia, y Sativanorte, Iza y Tota en Boyacá, por anomalías del sistema nervioso central, y Giraldo, Ciudad Bolivar, Liborina y Sopetrán en Antioquia, por anomalías de origen cromosómico, por lo cual debe dárseles prioridad en la determinación de los riesgos y el desarrollo de programas preventivos.

\section{Agradecimientos}

Al Instituto Nacional de Salud y al Departamento Administrativo Nacional de Estadística (DANE) por facilitar las bases de datos para el desarrollo de la presente publicación. A Diana Valencia, investigadora asociada, y a Alejandro Azofeifa, exinvestigador asociado, de los Centers for Disease Control and Prevention (CDC), por sus aportes durante el análisis de la información y la escritura del artículo. A Noël C. Barengo de The Task Force for Global Health, por sus aportes al diseño y la revisión del manuscrito, así como a la redacción del resumen en inglés.

\section{Conflicto de intereses}

Los autores declaran que no tienen ninguna relación de carácter financiero con ninguna organización o entidad que pudiera dar lugar a un conflicto de intereses. El contenido de esta publicación es de responsabilidad exclusiva de sus autores y no representa necesariamente la opinión del Task Force for Global Health, Inc., de TEPHINET o de los CDC.

\section{Financiación}

El estudio recibió apoyo mediante una beca de los CDC a través de TEPHINET, programa del Task Force for Global Health, Inc.

\section{Referencias}

1. Barboza-Argüello M, Umaña-Solís LM. Análisis de diez años de registro de malformaciones congénitas en Costa Rica. Acta Méd Costarric. 2008;50:221-9.

2. Organización Mundial de la Salud. Anomalías congénitas. Nota descriptiva $N^{\circ} 370$. Fecha de consulta: 21 de mayo de 2013. Disponible en: http://www.who.int/mediacentre/ factsheets/fs370/es/index.html.

3. Zarante I, Castillo M, García N, Suárez F, Gutiérrez C, Umaña A. Análisis clínico epidemiológico de factores asociados a malformaciones congénitas ECLAMC - Hospital Universitario San Ignacio, junio-diciembre de 2001. Univ Med. 2002;43:121-7.

4. World Health Organization. Global health observatory data repository. Number of deaths: WORLD by cause. Fecha de consulta: 21 de mayo de 2013. Disponible en: http://apps. who.int/gho/data/node.main.CODWORLD?lang=en.

5. Organización Panamericana de la Salud. Observatorio Regional en Salud - mortalidad. Fecha de consulta: 22 de mayo de 2013. Disponible en: http://ais.paho.org/phip/viz/ mort_causasprincipales_It_oms.asp.

6. Ministerio de la Protección Social. Indicadores básicos 2010. Situación de salud en Colombia. Fecha de consulta: 16 de marzo de 2012. Disponible en: https://www.minsalud. gov.co/sites/rid/Lists/BibliotecaDigital/RIDE/VS/ED/PSP/ Indicadores-basicos-en-salud-2010.pdf.

7. Ministerio de la Protección Social. Indicadores básicos 2011. Situación de salud en Colombia. Fecha de consulta: 10 de agosto de 2014. Disponible en: https://www.minsalud. gov.co/sites/rid/Lists/BibliotecaDigital/RIDE/VS/ED/PSP/ Indicadores-basicos-en-salud-2011.pdf.

8 Departamento Administrativo Nacional de Estadistica. Estadísticas vitales EEVV. Bogotá: DANE; 2009.

9. Embajada de Colombia en Alemania. Colombia, climas y regiones. Fecha de consulta: 3 de octubre de 2014. Disponible en: http://www.botschaft-kolumbien.de/paginas/c_ colombia_clima.htm.

10. Toda Colombia. Regiones naturales de Colombia. Fecha de consulta: 3 de octubre de 2014. Disponible en: http:// www.todacolombia.com/geografia/regionesnaturales.html.

11. Malagón D. Instituto Geográfico Agustín Codazzi. Los suelos de Colombia. Fecha de consulta: 24 de abril de 2015. Disponible en: http://www.sogeocol.edu.co/ documentos/05loss.pdf

12. Zuleta J, Gómez J, Jaramillo D. Análisis poblacional de la mortalidad perinatal. Medellín: Universidad de Antioquia; 2004. p. 115-33.

13. Snijders RJ, Sebire NJ, Nicolaides KH. Maternal age and gestational age-specific risk for chromosomal defects. Fetal Diagn Ther. 1995;10:356-67. http://dx.doi. org/10.1002/uog.128

14. Reefhuis J, Honein MA. Maternal age and nonchromosomal birth defects, Atlanta--1968-2000: Teenager or thirty-something, who is at risk? Birth Defects Res A Clin Mol Teratol. 2004;70:572-9. http://dx.doi.org/10.1002/ bdra.20065 
15. Chen XK, Wen SW, Fleming N, Yang Q, Walker MC. Teenage pregnancy and congenital anomalies: Which system is vulnerable? Hum Reprod. 2007;22:1730-5. http:// dx.doi.org/10.1093/humrep/dem043

16. Gordon A, Raynes C. Risk factors for antepartum stillbirth and the influence of maternal age in New South Wales, Australia: A population-based study. BMC Pregnancy Childbirth. 2013;13:12. http://dx.doi.org/10.1186/1471-239313-12

17. Lee K, Khoshnood B, Chen L, Wall SN, Cromie WJ, Mittendorf RL. Infant mortality from congenital malformations in the United States, 1970-1997. Obs Gynecol. 2001;98:620-7.

18. Departamento Administrativo Nacional de Estadística. Población y demografía. Fecha de consulta: 18 de enero de 2013. Disponible en: http://www.dane.gov.co/index.php/ poblacion-y-demografia/nacimientos-y-defunciones.

19. Nazer J, Cifuentes L, Águila A, Ureta P, Bello MP, Correa F, et al. Edad materna y malformaciones congénitas: un registro de 35 años. 1970-2005. Rev Méd Chile. 2007;135:1463-9. http://dx.doi.org/10.4067/S003498872007001100014

20. Hoffman Jl. Incidence of congenital heart disease: II. Prenatal incidence. Pediatr Cardiol. 1995;16:155-65. http:// dx.doi.org/10.1007/BF00794186

21. Hoffman JI, Kaplan S. The incidence of congenital heart disease. J Am Coll Cardiol. 2002;39:1890-900. http://dx.doi. org/10.1016/S0735-1097(02)01886-7

22. Khoshnood B. Trends in prenatal diagnosis, pregnancy termination, and perinatal mortality of newborns with congenital heart disease in France, 1983-2000: A populationbased evaluation. Pediatrics. 2005;115:95-101.

23. Jaramillo G, Hernández A, Mosquera W, Durán A. Cardiopatías congénitas. Fecha de consulta: 4 de octubre de 2014. Disponible en: http://scc.org.co/wp-content/ uploads/2012/08/capitulo15.pdf.

24. Christianson A, Howson CP, Modell B. March of Dimes. Global Report on Birth Defects. Fecha de consulta: 19 de enero de 2015. Disponible en: http://www.marchofdimes. org/materials/global-report-on-birth-defects-the-hidden-tollof-dying-and-disabled-children-appendix-b.pdf.
25. Departamento Administrativo Nacional de Estadística. Defunciones no fetales. Fecha de consulta: 19 de enero de 2013. Disponible en: http://www.dane.gov.co/index.php/ poblacion-y-demografia/nacimientos-y-defunciones/118demograficas/estadisticas-vitales/2877-defunciones-nofetales.

26. Baltaxe E, Zarante I. Prevalencia de malformaciones cardíacas congénitas en 44.985 nacimientos en Colombia. Arch Cardiol Méx. 2006;76:263-8.

27. Zarante I, Franco L, López C, Fernández N. Frecuencia de malformaciones congénitas: evaluación y pronóstico de 52.744 nacimientos en tres ciudades colombianas. Biomédica. 2010;30:65-71. http://dx.doi.org/10.7705/ biomedica.v30i1.154

28. Valdés J, Canún S, Reyes AE, Navarrete E. Mortalidad por defectos en el cierre del tubo neural en menores de 5 años de edad en México de 1998 a 2006. Salud Pública Méx. 2010;52:341-9.

29. Williams JL, Abelman SM, Fassett EM, Stone CE, Petrini JR, Damus K et al. Health care provider knowledge and practices regarding folic acid, United States, 2002-2003. Matern Child Health J. 2006;10:S67-72. http://dx.doi.org/10. 1007/s10995-006-0088-9

30. Bronberga R, Alfaro E. Mortalidad infantil por anencefalia en la Argentina. Análisis espacial y temporal (1998-2007). Arch Argent Pediatr. 2011;109:117-23.

31. Blencowe H, Cousens S, Modell B, Lawn J. Folic acid to reduce neonatal mortality from neural tube disorders. Int J Epidemiol. 2010;39 (Suppl.1):i110-21. http://dx.doi. org/10.1093/ije/dyq028

32. República de Colombia. Decreto número 1944 de 1996 por el cual se reglamenta la fortificación de la harina de trigo y se establecen las condiciones de comercialización, rotulado, vigilancia y control (Octubre 28 de 1996). Diario Oficial No. 42909 del 30 de octubre de 1996.

33. Dolk H. What is the 'primary' prevention of congenital anomalies? Lancet. 2009;374:378. http://dx.doi.org/10.1016/ S0140-6736(09)61411-2

34. Dolk H. Epidemiologic approaches to identifying environmental causes of birth defects. Am J Med Genet C Semin Med Genet. 2004;125C:4-11. http://dx.doi.org/10.1002/ajmg. c. 30000 